\title{
Identification of Pesticide Transformation Products in Food by Liquid Chromatography/Time-of-Flight Mass Spectrometry via "Fragmentation- Degradation" Relationships
}

\author{
Juan F. García-Reyes ${ }^{1,2}$, Antonio Molina-Díaz ${ }^{1}$, Amadeo R. Fernández-Alba ${ }^{2 *}$ \\ SUPPLEMENT MATERIAL
}

Toxicity data available for the compounds studied is included in the following tables:

Table S1. Toxicity data available for amitraz and their transformation products

\begin{tabular}{|c|c|c|c|c|c|}
\hline $\begin{array}{l}\text { Toxicological } \\
\text { profile }\end{array}$ & Amitraz & $\begin{array}{l}\text { 2,4 dimethyl } \\
\text { N-methyl- } \\
\text { formamide }\end{array}$ & $\begin{array}{l}2,4 \\
\text { dimethylani } \\
\text { line }\end{array}$ & $\begin{array}{l}2,4 \\
\text { dimethylformamide }\end{array}$ & $\begin{array}{l}\text { N,N'-bis- } \\
\text { dimethylphenylform } \\
\text { amide }\end{array}$ \\
\hline $\begin{array}{l}\text { Pesticide } \\
\text { Action } \\
\text { Network } \\
\text { (PAN) Bad } \\
\text { Actors }\end{array}$ & $\begin{array}{l}\text { Category 1: } \\
\text { high toxicity }\end{array}$ & No available & No available & No available & No available \\
\hline $\begin{array}{l}\text { Central } \\
\text { Nervous } \\
\text { system } \\
\text { depression }\end{array}$ & $\begin{array}{l}\text { Yes, due xylene } \\
\text { group }(2,4- \\
\text { dimethylaniline })\end{array}$ & $\begin{array}{l}\text { Positive } \\
\text { Higher } \\
\text { toxicity than } \\
\text { Parent } \\
(2,4- \\
\text { dimethylanil } \\
\text { ine) moiety }\end{array}$ & $\begin{array}{l}\text { Possible } \\
(2,4- \\
\text { dimethylanil } \\
\text { ine) moiety }\end{array}$ & $\begin{array}{l}\text { Possible } \\
\text { (2,4-dimethylaniline) } \\
\text { moiety }\end{array}$ & $\begin{array}{l}\text { Possible } \\
(2,4 \text {-dimethylaniline }) \\
\text { moiety }\end{array}$ \\
\hline Acute toxicity & moderate & No available & No available & No available & No available \\
\hline Carcinogen & possible & & & & \\
\hline Genotoxicity & No & $\begin{array}{l}\text { Positive } \\
\text { Forward } \\
\text { mutation ( } \\
\text { Mouse } \\
\text { L5178Y } \\
\text { tk+l-) }\end{array}$ & No & No & No \\
\hline $\begin{array}{l}\text { Cholinesterase } \\
\text { inhibitor }\end{array}$ & No & No available & No available & No available & No available \\
\hline $\begin{array}{l}\text { Developmental } \\
\text { or } \\
\text { reproductive } \\
\text { toxin }\end{array}$ & High toxicity & No available & No available & No available & No available \\
\hline $\begin{array}{l}\text { Endocrine } \\
\text { disruption }\end{array}$ & $\begin{array}{l}\text { No available } \\
\text { weight of } \\
\text { evidence } \\
\text { summary } \\
\text { assessment }\end{array}$ & No available & No available & No available & No available \\
\hline
\end{tabular}

From: Joint Meeting on Pesticide Residues (JMPR) Evaluations 1998, Part II Toxicological) 
Table S2. Toxicity data available for malathion and their transformation products

\begin{tabular}{|c|c|c|c|c|}
\hline $\begin{array}{l}\text { Toxicological } \\
\text { profile }\end{array}$ & Malathion & $\begin{array}{l}\text { Desmethylmalathion } \\
\text { (TP-2, m/z 317) }\end{array}$ & $\begin{array}{l}\mathrm{m} / \mathrm{z} 303 \\
\text { TP-2 }\end{array}$ & IsoMalathion \\
\hline $\begin{array}{l}\text { Pesticide Action } \\
\text { Network (PAN) Bad } \\
\text { Actors }\end{array}$ & $\begin{array}{l}\text { Category 1: } \\
\text { high toxicity }\end{array}$ & No available & No available & Category 1 : high toxicity \\
\hline Acute toxicity & moderate & No available & No available & $\begin{array}{l}\text { No available weight of } \\
\text { evidence summary } \\
\text { assessment }\end{array}$ \\
\hline Carcinogen & possible & & & $\begin{array}{l}\text { No available weight of } \\
\text { evidence summary } \\
\text { assessment }\end{array}$ \\
\hline $\begin{array}{l}\text { Cholinesterase } \\
\text { inhibitor }\end{array}$ & High activity & No available & No available & High activity \\
\hline $\begin{array}{l}\text { Developmental or } \\
\text { reproductive toxin }\end{array}$ & possible & No available & No available & $\begin{array}{l}\text { No available weight of } \\
\text { evidence summary } \\
\text { assessment }\end{array}$ \\
\hline $\begin{array}{l}\text { Endocrine } \\
\text { disruption }\end{array}$ & suspected & No available & No available & $\begin{array}{l}\text { No available weight of } \\
\text { evidence summary } \\
\text { assessment }\end{array}$ \\
\hline
\end{tabular}

From: Joint Meeting on Pesticide Residues (JMPR) Evaluations 1998, Part II Toxicological)

\footnotetext{
${ }^{1}$ Department of Physical and Analytical Chemistry, University of Jaén, 23071 Jaén, Spain

${ }^{2}$ Pesticide Residue Research Group, Department of Hydrogeology and Analytical Chemistry, University of Almería, 04120 La Cañada de San Urbano, Almería, Spain
}

*Corresponding author: Amadeo R. Fernández-Alba. Pesticide Residue Research Group, Department of Hydrogeology and Analytical Chemistry, University of Almeria, 04120 La Cañada de San Urbano (Almería), Spain; Tel.: (+34)950015034; Fax: (+34)950014102; E-mail: amadeo@ual.es 